\title{
Prototype of a novel MEA bioreactor measuring neural network activity continuously over long periods
}

\author{
E. Biffi ${ }^{1}$, A. Pedrocchi ${ }^{1}$, A. Menegon ${ }^{2}$, D. Ghezzi ${ }^{3}$, G.B. Fiore ${ }^{1}$, G. Ferrigno ${ }^{1}$ \\ (1) Bioengineering Department, Politecnico di Milano, Milano, Italy \\ (2) R\&D Laboratory, Alembic, San Raffaele Scientific Institute, Milano, Italy \\ (3) Italian Institute of Technology, Genova, Italy
}

\section{Background and Aims}

Spontaneously active neuronal networks show a high degree of sensitivity to subtle changes in physical and chemical environment of the culture [Gross et al., 1994]. Applications involving Micro Electrode Arrays (MEAs) in long-term analysis suffer from some limits (i.e. pH variation, sterility problems, medium evaporation) imposed by the standard experimental setup, causing a gradual decline in the health of these cultures. Since 1988, systems improving cells surviving were developed; temperature controlled bath chambers were designed demonstrating temperature dependence of neuronal properties [Forsythe et al., 1988; Toyotomi et al., 1989]. Sterility tools [Potter et al., 2001] and systems combining electrical recording and perfusion [Mukai et al., 2003] were considered to overcome some of the above-mentioned limits. Controlled medium environment [Gross et al. 1994] and miniaturized and portable chambers [Blau et al., 2001, Pancrazio et al., 2003] represented important steps in literature. However, none of the previous mentioned systems fulfilled completely this issue. In this work we present the technological development of an experimental system to measure neuronal network activity with MEAs continuously over long periods in a controlled atmosphere; our aim is to provide a single tool that record and process on-line neuronal action potentials without the need of an external incubator.

\section{Methods}

The incubating chamber prototype was realized with plates of polymethylmethacrylate (PMMA). The sizes and shape were designed to be suitable for cells monitoring on an inverted microscope. The MEA housing was designed with Pro-Engineer Wildfire and manufactured using subtractive rapid prototyping (Roland Modela MDX-40). The heating was obtained with a water bath surrounding the incubating chamber. Gasses and humidity control (95\% Air, 5\% $\mathrm{CO}_{2} ; 95 \%$ humidity) were developed using a commercial $\mathrm{CO}_{2}$ and bubbling module (Okolab s.r.l., Italy). Proper openings for medium and pharmacological agents insertion were obtained, assuring sterility with pierce silicone membranes for needles insertion. Pin connections for electrical signals acquisition were located through the top of the chamber; the electronic circuitry for the activity recording was placed on the superior external surface of the device. The whole system was sterilized with Ethylene Oxide (ETO). The device was tested in absence of cells to verify the maintenance of sterility and a preliminary validation on biological environment was performed. Software simulations and hardware feasibility study were achieved to design the on-line spike detection and clustering algorithm for future implementation on hardware and have been discussed in a previous paper [Biffi et al., 2010].

\section{Results}

First, the closed loop temperature control demonstrated to reach the steady-state temperature quite rapidly, maintaining it in the range of $37^{\circ} \mathrm{C}+/-1^{\circ} \mathrm{C}$. Preliminary prototype validation showed a good capability of cells growth and sterility preservation no less than 6 weeks. Concerning neuronal activity recordings, MEA's electrodes allowed to record signals without interference in the electrical measures. Finally, the chamber transparency allowed to observe networks on MEA biochip, with a 10x objective magnification on an inverted microscope (Axiovert 2000, Zeiss).

\section{Conclusion}

The bioreactor seemed to satisfy the imposed requirements, allowing long-term optical and electrophysiological monitoring of neuronal networks cultured on MEA biochips. An appropriate chamber could be very important for the successful of long experiments, playing a critical role in understanding some slow behaviours of a neuronal network, such as the long-term synaptic plasticity. 